\title{
Feedforward/Feedback Multivariable Control Design for High Speed Nanopositioning
}

\author{
Mohamed Kara Mohamed, William P. Heath and Alexander Lanzon
}

\begin{abstract}
This paper proposes a two degree of freedom control using a combined feedforward/feedback architecture for MIMO nanopositioning stages. The proposed control system provides higher bandwidth and better performance compared with a single degree of freedom feedback controller. The paper proposes a systematic synthesis methodology to design the controller based on closed loop performance. The results are verified via simulation and hardware experiment.
\end{abstract}

\section{INTRODUCTION}

Nanopositioning stages are used in a wide range of nanosciences and nanotechnologies, e.g., Atomic Force Microscopes (AFMs), Scanning Tunnelling Microscopes (STMs), lithography tools and molecular biology [1]. These stages are typically flexible structures driven by piezoelectric actuators and the position is measured by capacitive sensors allowing high precision. However, control of nanopositioning stages is technically challenging [2]. The dynamic control is limited by nonlinearities, power limitations and the many lightly damped resonances, whose frequencies vary with load. While stability for collocated nanopositioning stages in closed loop systems is analysed using several methods, e.g., the negative imaginary theory [3], [2], stages with noncollocated sensors and actuators have nonminimum phase dynamics [4]. In order to preserve robustness, controllers are typically designed with low bandwidth. This has a direct and detrimental effect on experiment time in all applications. Moreover, applications have recently emerged with high demands of increasing bandwidth and load requirements [4]. In response to these challenges, several feedback controllers have been introduced in the literature, e.g., [5], [6], [7] to damp the resonant peaks and increase the bandwidth. However, these methods are still limited by the well known limitations of feedback. Six fixed-structure feedback-based damping control techniques have been analysed and experimentally compared in [8], with a conclusion that the performance is fairly similar among these schemes.

The role of feedforward control in nanopositioning applications is reviewed in [4]. The implementation of model inversion-based feedforward control for specific applications of nanopositioning systems is considered in [9], [10], [11]. In these publications, high speed scanning has been achieved

\footnotetext{
*This work is funded by EPRSC under the grant EP/H016600/1.

The authors are with the Control Systems Centre, School of Electrical and Electronic Engineering, Sackville Street Building, University of Manchester, Manchester, M13 9PL, UK. mohamed.karamohamed@manchester.ac.uk

william.heath@manchester.ac.uk

alexander.lanzon@manchester.ac.uk
}

using model-inversion feedforward controllers only. However, feedforward controllers lack the necessary robustness when nonlinearities and model uncertainties exist, which is inevitable in nanopositioning stages.

In principle, a combination of feedforward and feedback controllers can be considered to improve performance and retain robustness. However, in the literature, no such control scheme is explored in depth for nanopositioning stages. Although, the authors in [12] have combined a PI feedback controller with a model-inverse based feedforward filter for an (AFM) application, the results are based on simulation study only and for a decoupled system.

The literature of nanopositioning systems is rich with SISO control design associated with single degree of freedom control structure. Multi-axis stages are commonly considered decoupled systems, and cross-coupling between channels is ignored ([13], [14], [15], [1] and the references therein). Behind this trend is the fact that the flexible structure of nanopositioning stages makes the coupling negligible when operating at very low speed [14]. While this assumption is valid for low frequency input commands, the coupling becomes more significant as the operational speed increases. The decoupling assumption becomes questionable when the operating bandwidth is close to the first resonant peak.

This paper considers a two degree of freedom control based on a feedforward/feedback combination structure for MIMO coupled nanopositioning stages. The proposed control system is a special case of the class discussed in [16] using coprime factorisation. The target is to increase the bandwidth of the closed loop system while preserving the loop robustness. The feedforward filter and reference filter are designed using an inner-outer factorisation of the stage's model together with a low pass filter. The feedback controller is synthesised using an $H_{\infty}$ loop shaping design method. In addition, a coupling index is used to measure the coupling of the closed loop system channels. The commercial stage NPS-XY-100A from Queensgate Inc. is used to test the effectiveness of the proposed control structure and analyse the results.

The paper begins with the design methodology in Section II. The control implementation to a nanopositioning stage is discussed in Section III. A summary of the discussion along with concluding remarks is provided in Section IV.

\section{The Design Methodology}

The proposed feedforward/feedback $(\mathrm{Ff} / \mathrm{Fb})$ control system is a two degree of freedom structure, where each part of the design contributes to the performance of the closed loop 


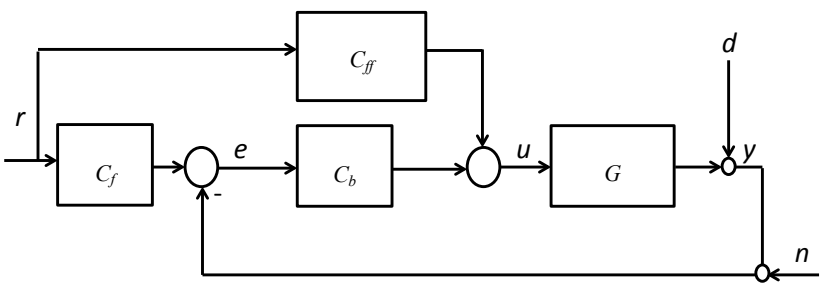

Fig. 1. Block Diagram of the proposed feedforward/feedback control scheme.

system. The tracking performance and the coupling index of the closed loop system are used to analyse the proposed control scheme and compare its performance with the performance of feedback-only controller. Fig. 1 represents the block diagram of the combined feedforward/feedback control scheme for MIMO stage systems. The nominal disturbanceto-output sensitivity transfer function $S_{d}$, which maps the disturbances $d$ to the output position $y$, is given by:

$$
S_{d}=\left(I+G C_{b}\right)^{-1} \text {. }
$$

The nominal noise-to-output transfer function $S_{n}$, which maps the sensor noise $n$ to the output position $y$, is given by:

$$
S_{n}=-\left(I+G C_{b}\right)^{-1} G C_{b} .
$$

The nominal reference-to-output tracking function is defined as the transfer function from the reference $r$ to the output $y$ and is given by:

$$
T_{r y}=\left(I+G C_{b}\right)^{-1} G\left(C_{b} C_{f}+C_{f f}\right),
$$

where $G$ is the model of the stage. The reference and feedforward filters are $C_{f}$ and $C_{f f}$ respectively, and $C_{b}$ is the feedback controller.

Eqs. (1) - (3) indicate that a good control scheme should make the reference-to-output transfer function $T_{r y}$ close to unity within the frequency region of the required bandwidth for good tracking. The disturbance-to-output sensitivity $S_{d}$ should be small at the same frequency region to minimise the effect of output disturbances on the output position. Furthermore, the noise-to-output transfer function $S_{n}$ should be small at high frequencies to avoid high frequency noise exciting unmodelled dynamics. These design criteria can be expressed explicitly using the frequency dependent singular values ${ }^{1}$ :

$$
\begin{aligned}
& \sigma_{\max }\left(S_{d}\right)_{\omega<\omega_{b}}<<1, \\
& \sigma_{\max }\left(S_{n}\right)_{\omega>\omega_{b}}<<1, \\
& \sigma_{\max }\left(T_{r y}\right)_{\omega<\omega_{b}} \approx 1, \quad \sigma_{\min }\left(T_{r y}\right)_{\omega<\omega_{b}} \approx 1,
\end{aligned}
$$

where $\omega_{b}$ is the designed bandwidth of the closed loop system and $\sigma_{\max (\min )}$ is the maximum (minimum) frequency dependent singular value of the related MIMO function.

For MIMO systems, it is also important to measure the coupling of the system. The target is always to have small

\footnotetext{
${ }^{1}$ As $\left|S_{d}\right|+\left|S_{n}\right|=I$, then both objectives in (4) and (5), which are in two different frequency regions, can be achieved by considering one function only which is usually $S_{n}$.
}

off-diagonal elements to minimise the effect of coupling on the closed loop system. In this paper, the coupling measure discussed in [17] is used to quantify the coupling among channels of the closed loop system. For a fully actuated $2 \times 2$ MIMO system, the full closed loop system from $r$ to $y$, can be written as:

$$
G_{c l}=\left[\begin{array}{ll}
g_{11} & g_{12} \\
g_{21} & g_{22}
\end{array}\right] .
$$

Then, the coupling between channels can be measured by defining the index $Q$ as:

$$
Q=\frac{\prod_{i \neq j} g_{i j}}{\prod_{i=j} g_{i j}} \quad i, j \in\{1,2\} .
$$

The index $Q$ is frequency dependent and for small coupling among channels, we should have:

$$
\sigma_{\max }(Q)_{\omega<\omega_{b}}<<1 .
$$

\section{A. Advantages and limitations}

Using the structure depicted in Fig. 1 and following the discussion of [18], the feedforward and reference filters should be designed respectively as $C_{f f}=M$ and $C_{f}=N$ for a coprime factorisation of $G=N M^{-1}$. In this case, the tracking performance of the loop becomes dependent only on $C_{f}$ where we have $T_{r y}=C_{f}=N$ for the nominal case. The feedback controller $C_{b}$ acts as an independent source of control effort to improve the robustness of the loop in terms of disturbance rejection and noise attenuation as specified in the Inequalities (4) and (5).

In this paper, the inner-outer factorisation is chosen for the design of $C_{f}$ and $C_{f f}$, where the dynamics of the stage model are inverted using the outer factor and applied to the reference signal to design $C_{f f}$, while $C_{f}$ is chosen to be the inner factor. By using inner-outer factorisation, the proposed design is a special case of the structures discussed in [18], [16] where the set-point is filtered by coprime factors of the plant before being injected into the loop (see Fig. 5 in [18]). The choice of inner-outer factorisation with a filter gives a useful intuitive feel for the frequency domain properties of the feedforward, nevertheless other choices, in particular normalised coprime factors of the plant's model, may be more advantageous and their role in the context of nanopositioning is the subject of current research. The feedback control is designed using $H_{\infty}$ loop shaping method to handle uncertainties and improve the robustness of the system. The design of $C_{f f}$ and $C_{f}$ for the case of inner-outer factorisation along with the design $H_{\infty}$ feedback controller will be discussed in detail in the next section.

The question that might be raised here is: can a feedbackonly controller be optimised to achieve the targets and hence no need is there for a two degree of freedom control structure? The answer is no, and the justification behind this answer lies in the fact that a feedback-only controller has limitations irrespective of the design method. These limitations become severe for lightly damped systems or in the presence of nonminimum phase zeros, which occurs in many nanopositioning stages. Generally speaking, the 
maximum closed-loop bandwidth is less than $2 \%$ of the first resonance frequency in nanopositioning systems when using only single degree of freedom feedback controller [5]. For more details on limitations of feedback for nanopositioning stages, see [5].

\section{Control Design AND Implementation}

This section presents the design and implementation of the proposed control system for the commercial nanopositioning system NPS-XY-100A. The model of the stage is identified first, and then the controller is designed and implemented in both simulation and hardware setup.

\section{A. System Identification}

The NPS-XY-100A stage is a nanopositioning system of two axes $x, y$. The supplied inputs to the system $u_{x}, u_{y}$ are the voltages for the piezoelectric actuators, and the outputs $y_{x}, y_{y}$ are the displacements along the two perpendicular axes, represented by the voltage measured from the capacitive sensors. The stage is driven by two independent electronic circuitry units (NPS2100). To obtain the MIMO (two-by-two) model of the stage over the frequency range of interest, a Pseudo-Random Binary Sequence (PRBS) signal of $0.4 \mathrm{~V}$ peak-to-peak magnitude is applied to each input independently and the outputs of both channels are measured. The signal is applied to the system and the output is measured via the NI DAQ card NIPCI-6154 with a sampling rate of $10 \mathrm{KHz}$. The signals are processed and the model is obtained using MATLAB System Identification Toolbox and utilising the subspace method. The reader should note that the frequency response of the system includes the dynamics of all electronic units and the driving computer for the NI card that exist in the loop. This information is important when interpreting the phase response of the system as in Fig. 3 and 4.

The measured frequency response of the stage shows a symmetrical structure with identical frequency response of both channels $x$ and $y$. In addition, the frequency response indicates a nonminimum phase lightly damped system with multi-mode resonance frequency. The nonminimum phase zeros can be interpreted in the light of sampling delay and the noncollocated geometry of the stage's sensors and actuators. The first resonant peak, identical for both channels, occurs in the region of $1-1.5 \mathrm{Krad} / \mathrm{s}$ with a dynamic range of about $10 \mathrm{~dB}$. Fig. 3 shows the frequency response of the system ${ }^{2}$ along with the fitted continuous time transfer function for the diagonal channels $x x, y y$. The fitted transfer function is biproper of order 30 and achieves a fitting accuracy of $96.21 \%$ to collected data with MSE of $2.123 \times 10^{-5}$. Making the fitted model bi-proper transfer function is to minimise the effect of the modes corresponding to frequencies that lie outside the frequency region of interest [19]. The effects of unmodelled delay can be seen in the phase at high frequency.

Fig. 4 depicts the coupling frequency response and the continuous time fitted transfer function for both channels,

\footnotetext{
${ }^{2}$ For simplicity of representation, all phase graphs in this paper have the phase response wrapped to be in the range $\left[-180^{\circ}, 180^{\circ}\right]$.
}

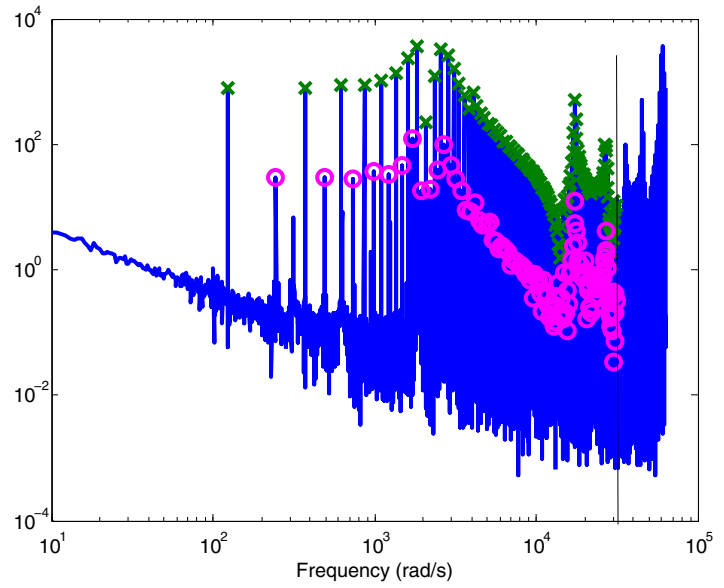

Fig. 2. Output spectrum of the excited channel response: the response at excited frequencies is marked by crosses; circles indicate nonlinearity since excitation at even harmonics is suppressed.

$G_{x y}=G_{y x}$. The fitted transfer function is of order 20 and achieves a fitting accuracy of $95.64 \%$ to collected data with MSE of $5.983 \times 10^{-06}$. The cross-coupling gain between the two channels, which is identical in both direction $x y$ and $y x$, is small at low frequencies $(\approx-40 \mathrm{~dB}$ in comparison with the excited channel $\approx 0 \mathrm{~dB}$ for the gain value at steady state), see Fig. 5. However, as the frequency increases, the cross-coupling becomes higher. In this paper, the frequency range of interest is up to the first resonance peak and the coupling effect should be considered.

The nonlinearity of the stage can be captured by plotting the Fourier Transform of the system response as shown in Fig. 2. The PRBS signal has a length of 255 samples repeated $n$ times to cover the length time of the experiment, with $n$ being an even number. Every other term is inverted so that the even harmonics are suppressed [20]. The relative excitation at these frequencies then indicates the level of nonlinearity. Although nonlinearities are present, the linear response is seen to dominate for the frequency range of interest, see Fig. 2, and hence in the sequel the system is assumed linear. The final model of the stage can be expressed by:

$$
G=\left[\begin{array}{ll}
G_{x x} & G_{y x} \\
G_{x y} & G_{y y}
\end{array}\right] .
$$

The next two subsections are dedicated to the control system design. It should be noted that each part of the control scheme, feedback and feedforward, is designed independently and therefore there is no restriction in designing any part before the other.

\section{B. Reference and Feedforward Control}

The reference and feedforward filters are designed via inner-outer factorisation and low pass filter. Given a transfer function matrix $G$, the inner-outer factorisation for the model of the system gives $G=G_{i} G_{o}$, where $G_{i}$ is a square, proper and inner while $G_{O}$ is a stable and minimum-phase. Broadly speaking, the inner-outer factorisation can be seen as minimum-phase approximation obtained by reflecting all 


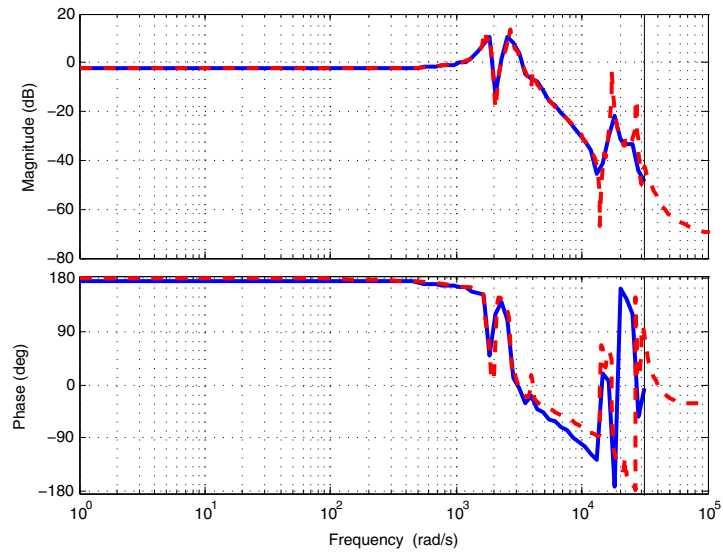

Fig. 3. Measured frequency response of the open loop diagonal channels, $x x=y y$, (solid line) and the fitted transfer function (dashed line).

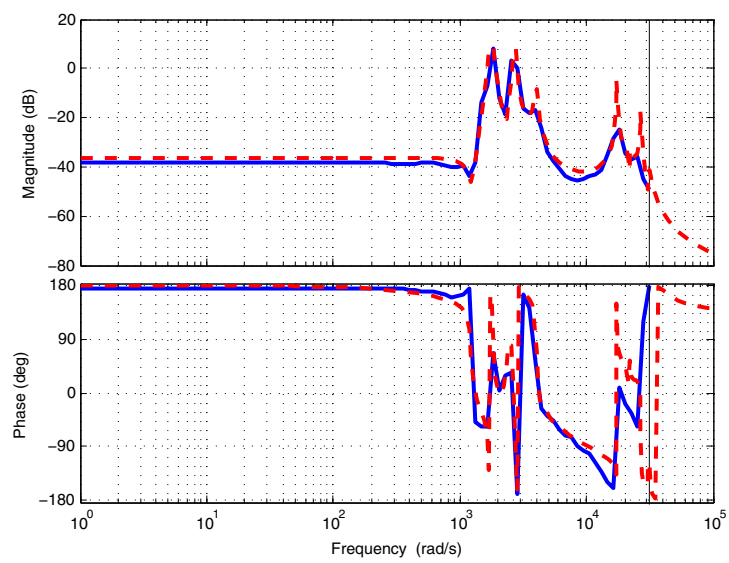

Fig. 4. Measured frequency response of the open loop cross-coupling channels, $x y=y x$, (solid line) and the fitted transfer function (dashed line).

unstable zeros into their mirrored stable zeros around the imaginary axis.

Both factors $G_{O}$ and $G_{i}$ include high frequency gain. However, the design of the reference and feedforward filters requires a limited bandwidth to avoid actuator saturation and undesirable dynamics. Hence, a low pass filter $P$ is needed to implement the reference and feedforward filters. The filter $P$ is given by:

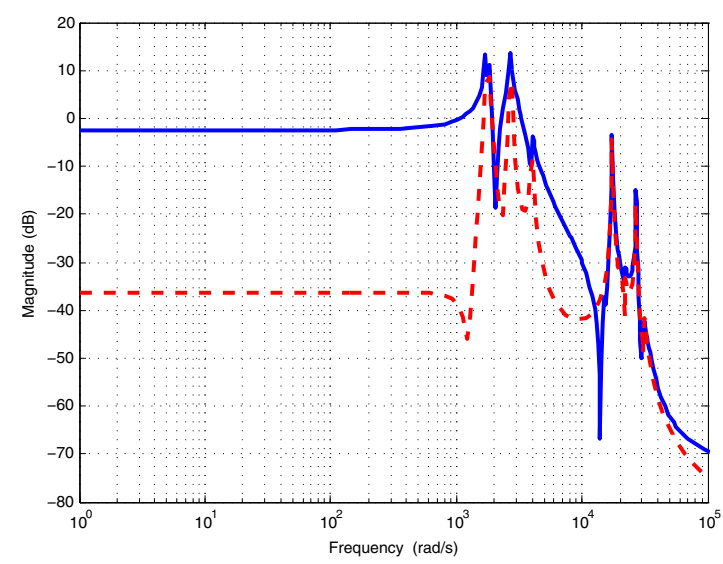

Fig. 5. Magnitude of frequency response for the diagonal channel (solid line) and the cross-coupling channel (dashed line).

$$
P=\left[\begin{array}{ll}
p & 0 \\
0 & p
\end{array}\right], \text { with } p=\frac{1}{(s / \alpha+1)^{\beta}}
$$

The parameter $\beta \geq 1$ and its value depends on the physical properties of the stage such as saturation limit and skew rate of the actuators. The parameter $\alpha>0$ is determined by the required bandwidth of the system. In our case, we choose $\alpha=1000 \mathrm{rad} / \mathrm{s}$ and $\beta=3$.

Then, the reference and feedforward filters are given by $C_{f}=G_{i} P$ and $C_{f f}=G_{o}^{-1} P$ respectively. Using this design, the tracking function $T_{r y}$, which specifics the tracking performance of the system, becomes independent from the feedback controller $T_{r y}=G_{i} P$ in the nominal case. This is a beneficial property of the proposed control architecture.

\section{Feedback Control}

In this paper, the feedback control $C_{b}$ is designed using $H_{\infty}$ loop shaping as proposed in [21]. The justification for the use of the $H_{\infty}$ loop shaping design method for nanopositioning MIMO systems and a discussion of its robustness properties can be found in [22] and [13]. The standard criteria reviewed in [23] to choose the weights for the loop shaping are followed here. The pre-compensator and post-compensator weights $W_{1}$ and $W_{2}$ are chosen to give the nominal system $G$ the desired open loop shape, and then a controller $K_{\infty}$ is synthesised to stabilise the shaped system $G_{s}=W_{2} G W_{1}$. Several methods have been proposed in the literature to facilitate the design of the weighting functions $W_{1}$ and $W_{2}$, see for example [24] and references therein.

Fig. 6 plots the singular values of the nominal stage model $G$ and the open loop shaped plant $G_{s}$. The shaping criteria are to have maximum bandwidth while fulfilling the following: $5 \%$ maximum overshoot, $0 \%$ steady state error, and $0.03 \mathrm{~nm}$ maximum RMS noise density on the output. The frequency response of the final implemented feedback controller is shown in Fig. 7 and its transfer function is $C_{b}=W_{1} K_{\infty} W_{2}$.

Finally, the coupling index $Q$ is defined as:

$$
Q=\frac{g_{x y} g_{y x}}{g_{x x} g_{y y}}
$$

where each $g_{i j}$ is the closed loop transfer function of the corresponding channel $i j$.

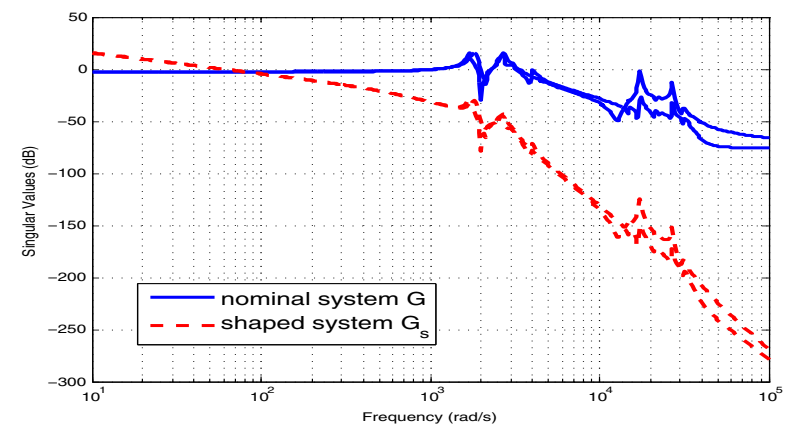

Fig. 6. Singular value plot of the nominal stage model $G$ (solid line) and the shaped system $G_{s}$ (dashed line). 


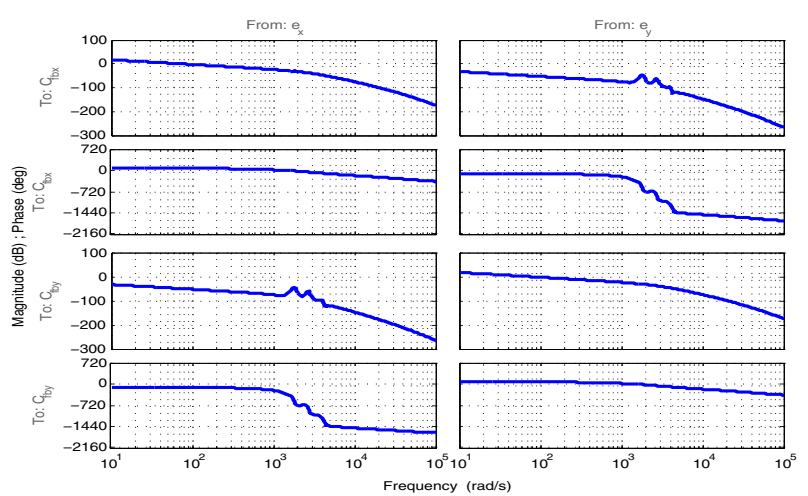

Fig. 7. Frequency response of the MIMO feedback controller $C_{b}$.

\section{Simulation Results}

This section reports the simulation results of the proposed combined feedforward/feedback control scheme. We use Matlab Simulink to obtain the results and we consider two scenarios; the proposed combined feedforward/feedback $(\mathrm{Ff} / \mathrm{Fb})$ as in Fig. 1 and the standard feedback only $(\mathrm{Fb})$ controller. Fig. 8 presents the command signal and the closed loop system response for a triangular input reference of $1 \mathrm{~V}$ peak-to-peak magnitude and $40 \mathrm{~Hz}$ frequency in $x$ channel for both scenarios. The results show that the case of $(\mathrm{Ff} / \mathrm{Fb})$ achieves better tracking compared with the cases of $(\mathrm{Fb})$. Taking into account the bandwidth of the system, the $(\mathrm{Ff} / \mathrm{Fb})$ structure also results in less coupling as will be seen in the discussion below for the coupling index.

Furthermore, the described performance measures in Section II are used for conclusive comparison. The singular value plots of the reference-to-output tracking function and the coupling index for both scenarios are depicted in Fig. 10. The figure reflects clearly that the combined $(\mathrm{Ff} / \mathrm{Fb})$ is superior to the $(\mathrm{Fb})$ for tracking with higher bandwidth. This means that the combined $(\mathrm{Ff} / \mathrm{Fb})$ control scheme achieves better tracking and higher bandwidth without losing the positive properties of the feedback control.

The $(\mathrm{Ff} / \mathrm{Fb})$ produces less coupling compared with the feedback only $(\mathrm{Fb})$ scenario, see the singular value plots of the coupling index in Fig. 10. It should be noted that the comparison becomes invalid beyond the bandwidth of the $(\mathrm{Fb}), 88 \mathrm{rad} / \mathrm{s}$, where the $(\mathrm{Fb})$ system loses input tracking and attenuates all signals beyond this frequency. Hence, to make comparison valid and useful, the coupling index is normalised and only plotted for the region of the bandwidth of interest.

\section{E. Experimental Results}

The designed control scheme is implemented to the NPSXY-100A stage using LabView RT module and the PZT actuators are driven by the NPS2100 NanoSensor single channel standalone module. The update rate of the controller is $8 \mathrm{KHz}$ and the response is measured using the NI DAQ card NIPCI-6154 with $8 \mathrm{KHz}$ sampling frequency. Fig 12 shows the system setup for the experiment.

Fig. 9 presents the response of the closed loop system to a $1 \mathrm{~V}$ peak-to-peak, $40 \mathrm{~Hz}$ triangular wave on $x$ channel.
As in the simulation case, two scenarios are considered: the feedforward/feedback $(\mathrm{Ff} / \mathrm{Fb})$ control scheme and the standard feedback only $(\mathrm{Fb})$ controller. The results match the simulation study where the feedforward/feedback control achieves better tracking with higher bandwidth. The reference-to-output tracking functions of the closed loop data are identified using an PRBS exciting signal. Then, the experimental coupling index is calculated using the identified transfer functions. Fig. 11 records the singular value plots of the reference-to-output tracking functions and the normalised coupling index functions respectively.
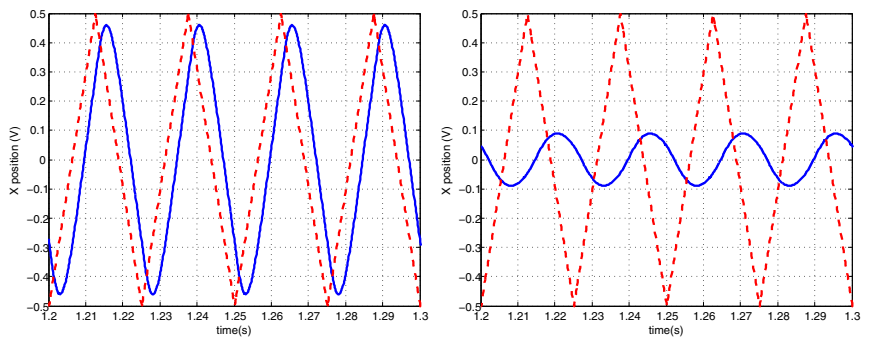

(a) The input signal (dashed line) and stage position (solid line) for the excited channel- $x$.
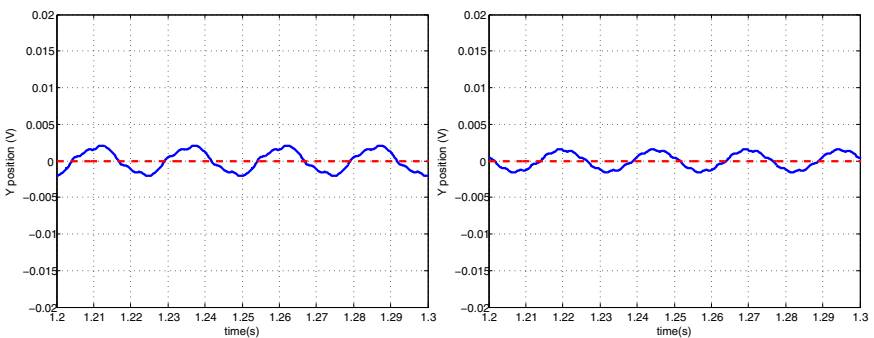

(b) The stage position (solid line) for the cross-coupling channel- $y$.

Fig. 8. Closed loop response to a $40 \mathrm{~Hz}$ triangle wave, $\mathrm{Ff} / \mathrm{Fb}$ (right) and Fb-only (left), simulation data
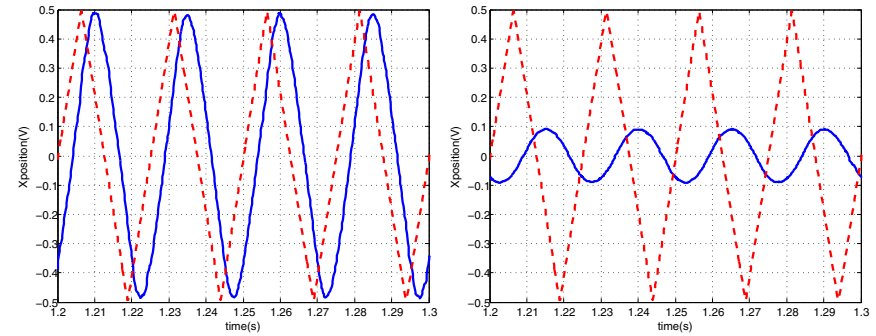

(a) The input signal (dashed line) and stage position (solid line) for the excited channel- $x$.
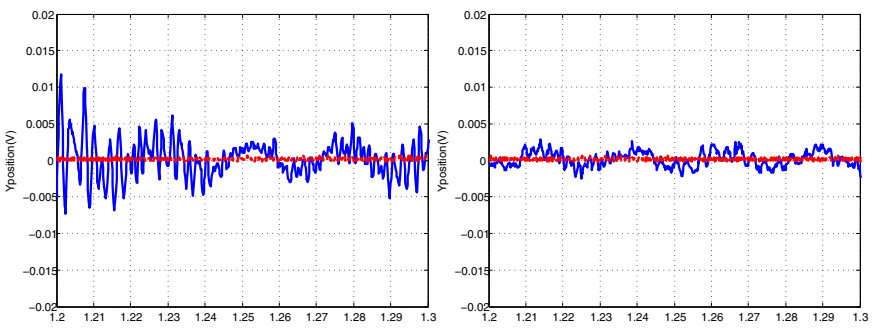

(b) The stage position (solid line) for the cross-coupling channel-y.

Fig. 9. Closed loop response to a $40 \mathrm{~Hz}$ triangle wave, $\mathrm{Ff} / \mathrm{Fb}$ (right) and $\mathrm{Fb}$ only (left), experimental data. 

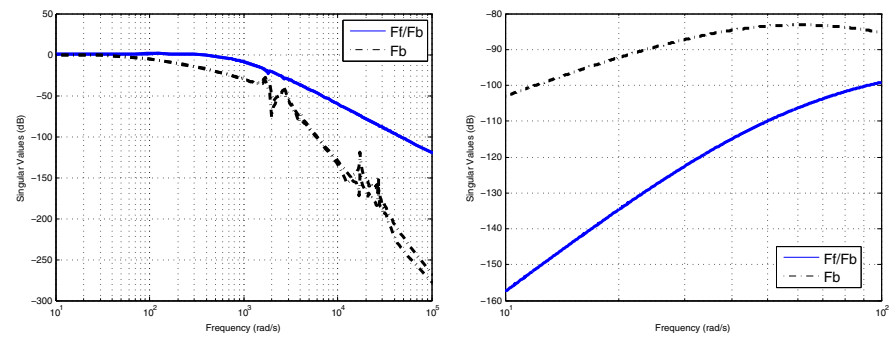

Fig. 10. The reference-to-output tracking transfer function (left) and coupling index (right) of the closed loop system, $\mathrm{Ff} / \mathrm{Fb}$ (solid line) and Fb-only (dashed line), simulation data.
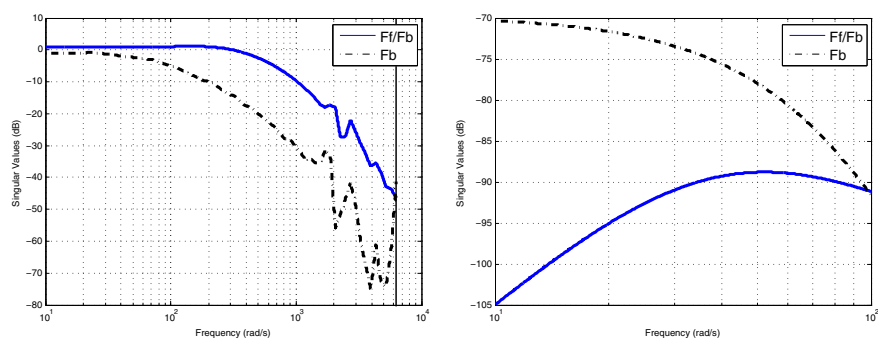

Fig. 11. The reference-to-output tracking transfer function (left) and coupling index (right) of the closed loop system, Ff/Fb (solid line) and Fb-only (dashed line), experimental data.

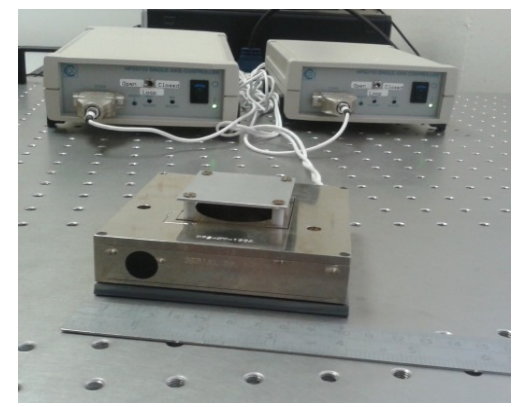

Fig. 12. Experimental hardware setup for controlling the NPS-XY-100A nanopositioning stage. The stage with the load adapter (front). The two NPS2100 NanoSensor single channel standalone modules (back).

\section{CONCLUSIONS}

This paper proposes a two degree of freedom MIMO feedforward/feedback control scheme for nanopositioning stages based on the structure given in [18]. Inner-outer factorisation is used as a special case of coprime factorisation to design the reference and feedforward filters. The feedback controller is synthesised using $H_{\infty}$ loop shape design method to gurnatee the robustness of the system. In the nominal case, the proposed $(\mathrm{Ff} / \mathrm{Fb})$ control overcomes the limitations of the feedback control systems and achieves better bandwidth without compromising the robustness property of feedback loop. Results are verified in both simulation and experimental studies for a commercial nanostage, NPS-XY-100A, from Queensgate Inc..

\section{REFERENCES}

[1] S. Devasia, E. Eleftheriou, and S. O. R. Moheimani, "A survey of control issues in nanopositioning," IEEE Transactions on Control Systems Technology, vol. 15, no. 5, pp. 802-823, 2007.
[2] I. R. Petersen and A. Lanzon, "Feedback control of negative-imaginary systems," IEEE Control Systems Magazine, vol. 30, no. 5, pp. 54-72, 2010.

[3] A. Lanzon and I. R. Petersen, "Stability robustness of a feedback interconnection of systems with negative imaginary frequency response," IEEE Transactions on Automatic Control, vol. 53, no. 4, pp. 10421046, 2008.

[4] G. M. Clayton, S. Tien, K. K. Leang, Q. Zou, and S. Devasia, "A review of feedforward control approaches in nanopositioning for highspeed SPM," Journal of Dynamic Systems, Measurement and Control, vol. 131, no. 6, pp. $061101-1-061$ 101-19, 2009.

[5] A. J. Fleming, "Nanopositioning system with force feedback for highperformance tracking and vibration control," IEEE/ASME Transactions on Mechatronics, vol. 15, no. 3, pp. 433-447, 2010.

[6] S. S. Aphale, A. J. Fleming, and S. O. R. Moheimani, "Integral resonant control of collocated smart structures," Smart Materials and Structures, vol. 16, no. 2, pp. 439-446, 2007.

[7] S. R. Moheimani and A. J. Fleming, Piezoelectric Transducers for Vibration Control and Damping, ser. Advances in Industrial Control. Springer, 2006.

[8] A. A. Eielsen, M. Vagia, J. T. Gravdahl, and K. Y. Pettersen, "Damping and tracking control schemes for nanopositioning," IEEE/ASME Transactions on Mechatronics, vol. 19, no. 2, pp. 432-444, 2014.

[9] B. Potsaid and J. Wen, "High performance motion tracking control," in Proceedings of the IEEE International Conference on Control Applications, vol. 1, 2004, pp. 718-723.

[10] D. Croft and S. Devasia, "Vibration compensation for high speed scanning tunneling microscopy," Review of Scientific Instruments, vol. 70, no. 12, pp. 4600-4605, 1999.

[11] G. Schitter, P. J. Thurner, and P. K. Hansma, "Design and inputshaping control of a novel scanner for high-speed atomic force microscopy," Mechatronics, vol. 18, no. 5, pp. 282-288, 2008.

[12] L. Y. Pao, J. Butterworth, and D. Abramovitch, "Combined feedforward/feedback control of atomic force microscopes," in Proceedings of The American Control Conference, 2007, pp. 3509-3515.

[13] A. Sebastian and S. Salapaka, "Design methodologies for robust nano-positioning," IEEE Transactions on Control Systems Technology,, vol. 13, no. 6, pp. 868-876, 2005.

[14] Y. Yong, K. Liu, and S. O. R. Moheimani, "Reducing cross-coupling in a compliant xy nanopositioner for fast and accurate raster scanning," IEEE Transactions on Control Systems Technology, vol. 18, no. 5, pp. $1172-1179,2010$

[15] Y. Yong, S. Aphale, and S. O. R. Moheimani, "Design, identification, and control of a flexure-based xy stage for fast nanoscale positioning," IEEE Transactions on Nanotechnology, vol. 8, no. 1, pp. 46-54, 2009.

[16] A. Dehghani, A. Lecchini-Visintini, A. Lanzon, and B. D. O. Anderson, "Validating controllers for internal stability utilizing closed-loop data," IEEE Transactions on Automatic Control, vol. 54, no. 11, pp. 2719-2725, 2009

[17] P. Grosdidier and M. Morari, "Interaction measures for systems under decentralized control," Automatica, vol. 22, no. 3, pp. 309-319, 1986.

[18] A. Lanzon, A. Lecchini, A. Dehghani, and B. D. O. Anderson, "Checking if controllers are stabilizing using closed-loop data," in Proceedings of The 45th IEEE Conference on Decision and Control, 2006, pp. 3660-3665.

[19] S. O. R. Moheimani and W. P. Heath, "Model correction for a class of spatio-temporal systems," Automatica, vol. 38, no. 1, pp. 147 - 155, 2002.

[20] K. R. Godfrey, A. H. Tan, H. A. Barker, and B. Chong, "A survey of readily accessible perturbation signals for system identification in the frequency domain," Control Engineering Practice, vol. 13, no. 11, pp. 1391-1402, 2005.

[21] D. McFarlane and K. Glover, "A loop shaping design procedure using synthesis," IEEE Transactions on Automatic Control, vol. 37, no. 6, pp. 759-769, 1992.

[22] S. Salapaka, A. Sebastian, J. P. Cleveland, and M. V. Salapaka, "High bandwidth nano-positioner: A robust control approach," Review of scientific instruments, vol. 73, no. 9, pp. 3232 - 3241, 2002.

[23] K. Zhou, J. C. Doyle, and K. Glover, Robust and Optimal Control. Prentice Hall New Jersey, 1996.

[24] M. Osinuga, S. Patra, and A. Lanzon, "An iterative algorithm for maximizing robust performance in $H_{\infty}$ loop-shaping design," International Journal of Robust and Nonlinear Control, vol. 23, no. 8, pp. 919-931, 2013 